\title{
Follow-up nationwide survey on predictive genetic testing for late-onset hereditary neurological diseases in Japan
}

\author{
Keiko Tanaka ${ }^{1}$, Yoshiki Sekijima ${ }^{2,3}$, Kunihiro Yoshida ${ }^{4}$, Mariko Tamai ${ }^{2}$, Tomoki Kosho ${ }^{2}$, Akihiro Sakurai ${ }^{1}$, \\ Keiko Wakui ${ }^{1}$, Shu-ichi Ikeda ${ }^{3}$ and Yoshimitsu Fukushima ${ }^{1,2}$
}

A follow-up nationwide survey on predictive genetic testing for late-onset neurological diseases in Japan was conducted. A questionnaire was sent to 89 institutional members of the Japan's National Liaison Council for Clinical Sections of Medical Genetics, and was returned by $60(67.4 \%)$. A total of 301 clients with an interest in predictive testing were accumulated from April 2006 to March 2011. The greatest interest was shown for spinocerebellar degeneration (SCD, $n=110$ ), followed by myotonic dystrophy type 1 (DM1, $n=69$ ), Huntington's disease (HD, $n=52$ ) and familial amyloid polyneuropathy (FAP, $n=35$ ). The ratios of clients who actually underwent predictive testing were: SCD, $21.8 \%$; DM1, 39.1\%; HD, 26.9\%; and FAP, $74.3 \%$, indicating that predictive testing was conducted very cautiously for untreatable neurological diseases in Japan. Clinical geneticists were predominantly involved in genetic counseling, whereas the participation of non-medical doctor (non-MD) staff, including nurses, clinical psychologists and genetic counselors, was not common. Lack of non-MD counseling staff was one of the most serious issues in conducting predictive testing, which has not been improved since the previous survey performed in 2006. Institutional arrangements, such as revision of medical insurance system regarding genetic testing and counseling, might be necessary to resolve this issue.

Journal of Human Genetics (2013) 58, 560-563; doi:10.1038/jhg.2013.34; published online 2 May 2013

Keywords: clinical geneticist; clinical psychologist; genetic counseling; genetic counselor; hereditary neurological diseases; predictive genetic testing

\section{INTRODUCTION}

In recent years, clinical sections of medical genetics and genetic counseling have been set up all over Japan. At present, there are 99 institutions, including all 80 university hospitals, with genetic counseling departments that have become members of the Japan's National Liaison Council for Clinical Sections of Medical Genetics (JNLCCSMG). The JNLCCSMG was established in 2003 for the purpose of cooperation between clinical sections of medical genetics in university hospitals and other core medical institutions (http://www.idenshiiryoubumon.org).

Predictive genetic testing is a matter of great concern for at-risk relatives of patients with late-onset hereditary neurological diseases, and is one of the most controversial topics in clinical genetics. In 2006, we performed the first nationwide survey on this issue in Japan; ${ }^{1}$ however, little is known about the global situations and problems regarding predictive testing after 2006. Here we report the results of a follow-up nationwide survey on predictive genetic testing for late-onset hereditary neurological diseases in Japan.

\section{MATERIALS AND METHODS}

A questionnaire was sent to 89 institutional members of the JNLCCSMG (all institutional members of the JNLCCSMG on 15 August 2011). The questionnaire contained seven questions as shown in Table 1. The chief person responsible for the genetic counseling department in each hospital was asked to fill out the questionnaire. This study was approved by the Ethical Committee of Shinshu University School of Medicine.

\section{RESULTS}

The questionnaire was returned by $67.4 \%(n=60)$ of the hospitals surveyed. Of the 60 hospitals, 41 (68.3\%) had clients with an interest in predictive testing for late-onset neurological diseases during the period from April 2006 to March 2011 (Table 1, Q1). Attitudes toward predictive testing for neurological diseases in each hospital were as follows: $22(36.7 \%)$ hospitals provide both genetic counseling and testing, 30 (50\%) hospitals provide genetic counseling only and $8(13.3 \%)$ hospitals do not provide genetic counseling or testing (Table 1, Q2).

${ }^{1}$ Department of Medical Genetics, Shinshu University School of Medicine, Matsumoto, Japan; ${ }^{2}$ Division of Clinical and Molecular Genetics, Shinshu University School of Medicine, Matsumoto, Japan; ${ }^{3}$ Department of Medicine (Neurology and Rheumatology), Shinshu University School of Medicine, Matsumoto, Japan and ${ }^{4}$ Department of Brain Disease Research, Shinshu University School of Medicine, Matsumoto, Japan

Correspondence: Dr Y Sekijima, Division of Clinical and Molecular Genetics, Shinshu University School of Medicine, 3-1-1 Asahi, Matsumoto 390-8621, Japan. E-mail: sekijima@shinshu-u.ac.jp

Received 6 December 2012; revised 3 April 2013; accepted 11 April 2013; published online 2 May 2013 
Table 1 Questionnaire and results regarding predictive genetic testing for hereditary late-onset neurological diseases

Q1 Have you had clients who visited your hospital with an interest in or requesting predictive genetic testing for late-onset neurological diseases during the period from April 2006 to March 2011? (total responding $=60$ )

Yes

No

Q2 Does your hospital provide genetic counseling and/or testing for late-onset neurological diseases? (total responding $=60$ )

Provides both genetic counseling and testing

Provides genetic counseling only

Does not provide genetic counseling or testing

Q3 What kinds of diseases were your clients interested in? Please indicate the numbers of families, clients, predictive tests performed and positive for disease-causing mutations for each disease (total responding $=41$ )

\begin{tabular}{|c|c|c|c|c|c|}
\hline Disease & $\begin{array}{c}\text { Number } \\
\text { of hospitals }\end{array}$ & $\begin{array}{l}\text { Number } \\
\text { of families }\end{array}$ & $\begin{array}{l}\text { Number } \\
\text { of clients }\end{array}$ & $\begin{array}{c}\text { Predictive } \\
\text { tests performed }\end{array}$ & $\begin{array}{c}\text { Positive for } \\
\text { disease-causing } \\
\text { mutations }\end{array}$ \\
\hline Spinocerebellar degeneration & 29 & 97 & 110 & $24(21.8 \%)$ & 8 \\
\hline Myotonic dystrophy type 1 & 21 & 59 & 69 & $27(39.1 \%)$ & 15 \\
\hline Huntington's disease & 22 & 46 & 52 & $14(26.9 \%)$ & 6 \\
\hline Familial amyloid polyneuropathy & 7 & 24 & 35 & $26(74.3 \%)$ & 10 \\
\hline Amyotrophic lateral sclerosis & 7 & 10 & 12 & $0(0 \%)$ & 0 \\
\hline Spinal and bulbar muscular atrophy & 6 & 9 & 9 & $0(0 \%)$ & 0 \\
\hline Alzheimer's disease & 3 & 4 & 4 & $1(25 \%)$ & 1 \\
\hline Facioscapulohumeral muscular dystrophy & 2 & 4 & 4 & $0(0 \%)$ & 0 \\
\hline Parkinson's disease & 1 & 1 & 3 & $0(0 \%)$ & 0 \\
\hline Adrenoleukodystrophy & 1 & 1 & 1 & $1(100 \%)$ & 0 \\
\hline Frontotemporal dementia and parkinsonism & 1 & 1 & 1 & $0(0 \%)$ & 0 \\
\hline Unknown & 1 & 1 & 1 & $0(0 \%)$ & 0 \\
\hline
\end{tabular}

Q4 How much did the following professions contribute to the counseling sessions of such clients? Please select the most appropriate percentage among the following: a: 0\%, b: 0-25\%, c: 25-50\%, d: 50-75\% and e: 75-100\% ('0\%' indicates no participation and ' $100 \%$ ' indicates full-time participation in the counseling sessions) (total responding $=48$ )

Clinical geneticist ${ }^{a}$

Neurologist

Psychiatrist

Nurse

Clinical psychologist

Genetic counselor ${ }^{\mathrm{b}}$

\begin{tabular}{ccccc}
$0 \%$ & $0-25 \%$ & $25-50 \%$ & $50-75 \%$ & $75-100 \%$ \\
\hline 2 & 4 & 4 & 9 & 29 \\
16 & 14 & 5 & 4 & 9 \\
40 & 8 & 0 & 0 & 0 \\
28 & 9 & 3 & 2 & 6 \\
32 & 6 & 3 & 2 & 5 \\
44 & 2 & 0 & 0 & 2
\end{tabular}

Q5 What is the reason for being unable to provide genetic counseling and/or testing? (multiple answers allowed) (total responding $=35$ )

Insufficiency of genetic counseling system in the hospital (go to Q6)

No clients interested in or requesting predictive genetic testing for neurological diseases (go to Q7)

Taking an opposing position on predictive genetic testing for late-onset, neurological diseases (go to Q7)

Q6 What kind of genetic counseling system is insufficient in your hospital? (multiple answers allowed) (total responding $=28$ )

Genetic counseling staff

Genetic counselorb

Clinical psychologist

Nurse

Clinical geneticist ${ }^{a}$

Psychiatrist

Neurologist

Medical social worker

Time for clinical practice of genetic counseling

Genetic testing system 
Q7 What do you think are the most important requirements for maintaining genetic counseling for such clients? Please select the three most important requirements from the following and rank them in the order of their importance, (1st) being most important (total responding $=60$ )

\begin{tabular}{|c|c|c|c|c|}
\hline & 1 st & 2nd & $3 r d$ & Rank unknown \\
\hline Genetic counseling staff & 28 & 9 & 8 & 6 \\
\hline Understanding and recognition of clinical genetics by the general & 10 & 5 & 9 & 3 \\
\hline Genetic testing system & 6 & 10 & 11 & 1 \\
\hline Administrative support for genetic counseling & 4 & 8 & 5 & 3 \\
\hline Time and space for clinical practice of genetic counseling & 2 & 14 & 12 & 4 \\
\hline Cooperation with the other hospitals & 2 & 4 & 2 & 4 \\
\hline Others & 1 & 2 & 2 & 0 \\
\hline
\end{tabular}

aClinical geneticist indicates a medical doctor who has the 'Japanese Board of Medical Genetics, Clinical Geneticist' certification.

'Genetic counselor indicates a non-medical doctor who has the 'Japanese Board of Medical Genetics, Certified Genetic Counselor' certification. The certificates are given by the Japan Society of Human Genetics and the Japanese Society for Genetic Counseling.

In total, 301 clients from 257 families were reported from the 41 hospitals during the study period. The greatest interest was shown with regard to SCD (97 families, 110 clients), followed by DM1 (59 families, 69 clients), HD (46 families, 52 clients) and FAP (24 families, 35 clients). The remaining clients were interested in amyotrophic lateral sclerosis, spinal and bulbar muscular atrophy, Alzheimer's disease, facioscapulohumeral muscular dystrophy, Parkinson's disease, adrenoleukodystrophy and frontotemporal dementia and parkinsonism. The numbers of clients who actually underwent predictive genetic testing were as follows: SCD, 24 (21.8\%); DM1, 27 (39.1\%); HD, 14 (26.9\%); FAP, 26 (74.3\%); Alzheimer's disease, 1 (25\%); and adrenoleukodystrophy, 1 (100\%). No clients interested in amyotrophic lateral sclerosis, spinal and bulbar muscular atrophy, facioscapulohumeral muscular dystrophy, Parkinson's disease and frontotemporal dementia and parkinsonism underwent predictive genetic testing (Table 1, Q3).

During the period of the survey, clinical geneticists were predominantly involved in genetic counseling, as they participated in $\geqslant 50 \%$ of the total counseling sessions in 38 of the 48 hospitals (79.2\%). Neurologists participated to a certain degree in counseling sessions in 32 hospitals $(66.7 \%)$. The contribution of psychiatrists was much smaller, as they participated in counseling sessions only in eight hospitals $(16.7 \%)$. The participation of non-medical doctor (nonMD) staff was not common, as nurses, clinical psychologists and genetic counselors were involved in $20(41.6 \%), 16(33.3 \%)$ and 16 (33.3\%) hospitals, respectively (Table 1, Q4).

Insufficiency of genetic counseling system, especially the lack of non-MD counseling staff, was the most serious obstacle to genetic counseling and/or testing in most hospitals (Table 1, Q5-7).

\section{DISCUSSION}

In a previous nationwide survey in Japan, ${ }^{1} 322$ clients (excluding FAP) visited hospitals with an interest in predictive testing for lateonset neurological diseases between April 2004 and May 2006 (161 clients per year). Surprisingly, the number of clients excluding FAP substantially decreased between April 2006 and March 2011, as only 266 clients visited the genetic counseling department (53 clients per year). A possible explanation for the decrease is that a large number of university hospitals and other core medical institutions had opened clinical sections of medical genetics in the early 2000s and clients who had been seeking predictive testing visited hospitals between 2004 and 2006 .

In the present study, the ratio of predictive genetic test usage was surveyed for the first time in Japan. Attitude toward predictive testing varies among counties ${ }^{2-12}$ because of culture, nationality, religion and availability of genetic counseling and testing systems. In addition, decision-making by clients in predictive genetic testing is largely influenced by their counselors ${ }^{7}$. In Japan, the ratios of predictive test usage in HD $(26.9 \%)$ and SCD $(21.8 \%)$ were much lower than those in other countries $\left(36-88 \%\right.$ for $\mathrm{HD}^{2-11}$ and $48-87 \%$ for $\mathrm{SCD}^{2,6,11,12}$ ), indicating that Japanese counselors and clients took a cautious approach toward predictive testing in untreatable neurological diseases. In addition to the Japanese culture and nationality, an insufficient psychological support system after predictive testing is considered to be an important reason for the low ratio of predictive test usage in Japan. In contrast to HD and SCD, most (74.3\%) clients interested in FAP actually underwent predictive testing in Japan, suggesting that the availability of disease-modifying therapy promoted the usage of testing. ${ }^{13-15}$

The present study showed that the respondents felt lack of non-MD counseling staff was one of the most serious issues in providing predictive testing for neurological diseases in Japan. This issue was also noted in the previous survey; ${ }^{1}$ however, the situation has not improved since then. Institutional arrangements, such as revision of the medical insurance system regarding genetic counseling and testing, might be necessary to resolve this issue.

There are two limitations that need to be acknowledged regarding the present study. The first limitation concerns the attitude to predictive testing. Both counselor's and client's attitude to predictive testing are influenced by various factors, such as age, sex, family composition, educational background, disease type and religion. However, we did not survey such factors in this study. The second limitation is that we did not survey actual hereditary risk in each client. These issues need to be analyzed in future follow-up studies.

\section{CONFLICT OF INTEREST}

The authors declare no conflicts of interest.

\section{ACKNOWLEDGEMENTS}

We are grateful to the respondents who participated in this study. This work was supported by a Grant-in-aid for Scientific Research (23591237 to YS), Health and Labour Sciences Research Grants for intractable diseases (12103066) and a Grant-in-Aid for Scientific Research (KAKENHI 23613004).

1 Yoshida, K., Wada, T., Sakurai, A., Wakui, K., Ikeda, S. \& Fukushima, Y. Nationwide survey on predictive genetic testing for late-onset, incurable neurological diseases in Japan. J. Hum. Genet. 52, 675-679 (2007).

2 Mariotti, C., Ferruta, A., Gellera, C., Nespolo, C., Fancellu, R., Genitrini, S. et al. Predictive genetic tests in neurodegenerative disorders: a methodological approach integrating psychological counseling for at-risk individuals and referring clinicians. Eur. Neurol. 64, 33-41 (2010). 
3 Cannella, M., Simonelli, M., D’alessio, C., Pierelli, F., Ruggieri, S. \& Squitieri, F. Presymptomatic tests in Huntington's disease and dominant ataxias. Neurol. Sci. 22, 55-56 (2001).

4 Mandich, P., Jacopini, G., Di Maria, E., Sabbadini, G., Abbruzzese, G., Chimirri, F. et al. Predictive testing for Huntington's disease: ten years' experience in two Italian centres. Ital. J. Neurol. Sci. 19, 68-74 (1998).

5 Gargiulo, M., Lejeune, S., Tanguy, M. L., Lahlou-Laforet, K., Faudet, A., Cohen, D. et al. Long-term outcome of presymptomatic testing in Huntington disease. Eur. J. Hum. Genet. 17, 165-171 (2009).

6 Goizet, C., Lesca, G. \& Durr, A. Presymptomatic testing in Huntington's disease and autosomal dominant cerebellar ataxias. Neurology 59, 1330-1336 (2002).

7 Bernhardt, C., Schwan, A. M., Kraus, P., Epplen, J. T. \& Kunstmann, E. Decreasing uptake of predictive testing for Huntington's disease in a German centre: 12 years' experience (1993-2004). Eur. J. Hum. Genet. 17, 295-300 (2009).

8 Maat-Kievit, A., Vegter-Van Der Vlis, M., Zoeteweij, M., Losekoot, M., Van Haeringen, A. \& Roos, R. Paradox of a better test for Huntington's disease. J. Neurol. Neurosurg. Psychiatry. 69, 579-583 (2000).

9 Dufrasne, S., Roy, M., Galvez, M. \& Rosenblatt, D. S. Experience over fifteen years with a protocol for predictive testing for Huntington disease. Mol. Genet. Metab. 102, 494-504 (2011).
10 Alonso, M. E., Ochoa, A., Sosa, A. L., Rodriguez, Y., Chavez, M., Boll, C. et al. Presymptomatic diagnosis in Huntington's disease: the Mexican experience. Genet. Test Mol. Biomarkers 13, 717-720 (2009).

11 Rodrigues, C. S., De Oliveira, V. Z., Camargo, G., Osorio, C. M., De Castilhos, R. M., Saraiva-Pereira, M. L. et al. Presymptomatic testing for neurogenetic diseases in Brazil: assessing who seeks and who follows through with testing. J. Genet. Couns. 21, 101-112 (2012).

12 Paneque, M., Lemos, C., Escalona, K., Prieto, L., Reynaldo, R., Velazquez, M. et al. Psychological follow-up of presymptomatic genetic testing for spinocerebellar ataxia type 2 (SCA2) in Cuba. J. Genet. Couns. 16, 469-479 (2007).

13 Takei, Y., Ikeda, S., Ikegami, T., Hashikura, Y., Miyagawa, S. \& Ando, Y. Ten years of experience with liver transplantation for familial amyloid polyneuropathy in Japan: outcomes of living donor liver transplantations. Intern. Med. 44, 1151-1156 (2005).

14 Sekijima, Y., Dendle, M. A. \& Kelly, J. W. Orally administered diflunisal stabilizes transthyretin against dissociation required for amyloidogenesis. Amyloid 13, 236-249 (2006).

15 Tojo, K., Sekijima, Y., Kelly, J. W. \& Ikeda, S. I. Diflunisal stabilizes familial amyloid polyneuropathy-associated transthyretin variant tetramers in serum against dissociation required for amyloidogenesis. Neurosci. Res. 56, 441-449 (2006). 